\title{
Frequency of HLA-B27 in Patients With Conductive System Disturbance and Implanted Permanent Pacemaker in Iran
}

\author{
Sima Sayah ${ }^{1}$, Morteza Ebrahimi ${ }^{1}$, Poorya Piroozmand ${ }^{1, *}$ \\ ${ }^{1}$ Department of Cardiology, Bouali Hospital, Qazvin University of Medical \\ Sciences, Qazvin, Iran \\ * Corresponding author: Poorya Piroozmand, Department of Cardiology, Bouali \\ Hospital, Qazvin University of Medical Sciences, Qazvin, Iran.E-mail: poorya_ \\ piroozmand@yahoo.com
}

DOI: $10.21859 / \mathrm{ijcp}-020103$

\begin{tabular}{l} 
Submited: 10.01 .2016 \\
Accepted: 12.13 .2016 \\
\hline
\end{tabular}

Keywords:

Aortic Valve Insufficiency

Atrioventricular Block

Pacemaker

Artificial

HLA-B27 Antigen

(C) 2016. International Journal of Cardiovascular Practice.

\begin{abstract}
Introduction: HLA-B27 is considered as one of the causes of heart conductive disorders. We studied the frequency of HLA-B27 among Iranian patients who had undergone permanent pacemaker implantation.

Methods: This descriptive and analytical study was performed among patients who underwent permanent pacemaker implantation. The questionnaire contains demographic data, underling diseases, and history of heart disease or heart surgery. The type of conductive heart disorder was mentioned there, too. We determined the regurgitation of aorta valve and its severity. Finally, HLA-B27 was accessed.All the data was entered in SPSS software and analyzed.

Results: From a total of 103 patients entered in this study, only 6 (5.8\%) were HLAB27-positive. The disturbance of conductive heart disorders based on the HLA-B27 positive or negative patients was not statistically different. Patients with HLA-B27 mostly had regurgitation of the aorta valve.

Conclusions: We concluded that the frequency of positive HLA-B27 genotype in patients with permanent pacemakers did not have any significant difference with the ones with negative HLA-B27 genotype. However; this genotype was associated with regurgitation of the aorta valve.
\end{abstract}

\section{INTRODUCTION}

Human leukocyte antigen (HLA) is a gene complex on the 6th chromosome which has several classes including the HLA-B27. HLA-B27 has a major role in susceptibility of a person to a number of autoimmune diseases $[1,2]$. The prevalence of this gene in the general population in Iran and the Middle East has been reported $8 \%$ and 3-5\%, respectively [35].

The association of HLA with pathogenesis of ankylosing spondylitis (AS) is well known. The role of HLA-B27 in the pathogenesis of AS and higher cardiovascular mortality in this disease have increased the efforts to determine the role of this antigen in cardiac diseases. The results of these researches have shown that a variety of cardiovascular problems are directly associated with HLA-B27. Aortic root dilation, aortic valve and mitral valve regurgitation, myocarditis, heart failure, pericarditis, pericardial effusion, atrioventricular (AV) node conduction block, and J-wave syndrome are some of these diseases $[6,7]$. On the other hand, electrophysiologic studies showed that conduction disorders in the AV node are more prevalent in carriers of HLA-B27 [6].

In the current study we aimed to evaluate the association between the AV node blocks and the presence of HLA-B27 in Iranian patients undergoing permanent pacemaker implan- tation with regard to lesser prevalence of this antigen in the Iranian society compared with European countries and the United States population [5].

\section{METHODS}

This cross-sectional prospective study was approved by Qazvin University of Medical Sciences Ethical Committee and was conducted by the Cardiology Department of Booali-Sina Hospital, Qazvin, from January 2014 to January 2015. All the patients who were candidates for permanent pacemaker implantation according to American College of Cardiology/ American Heart Association (ACC-AHA) guidelines were enrolled in this study. Patients with iatrogenic atrioventricular blocks such as post-electrophysiological study and catheter ablation or post-cardiac surgery were excluded. Patients who were on AV nodal blocking agents were included in this study if the AV conductions were impaired after discontinuation of the drug for at least five half-lives.

All the patients were informed about the study protocol and then provided a written informed consent. Venous blood sample was used for the detection of HLA-B27 antigen in all the included patients. Patients were divided into two groups according to presence of HLA-B27.

Data were analyzed using SPSS 16.0 for Windows (SPSS Inc. 
Chicago, IL, USA) and were presented as numbers (frequency). Chi-squared test, Fisher's exact and Mann-Whitney U were used to analyze the qualitative variables between the two groups. $\mathrm{P}<0.05$ was considered as the significance level between the treatments.

\section{RESULTS}

In this study 103 patients were evaluated. Demographic features are shown in Table 1.

Complete heart block was observed in 72 patients (69.9\%); 9 (8.7\%) patients had second-degree AV block and $4(6.8 \%)$ had sick sinus syndrome. Simultaneous involvement of AV node and SA node was seen in four pa- tients. Other kinds of AV block including prolonged HV interval were the indications of pacemaker implantation in 11 patients.

There was no significant difference in the indications of pacemaker implantation between male and female patients.

HLA-B27 was positive in only $6(5.8 \%)$ patients. Fifty percent of them had complete heart blocks. Distributions of this antigen based on the variety of conduction disturbances are shown in Table 2. Although HLA-B27 was more positive in male patients, the difference between the two genders was not significant.

Aortic valve regurgitation was more prevalent in patients with positive HLA-B27 compared with the negative group (5 (83.3\%) vs. $39(40.2 \%)$ respectively, $\mathrm{P}=0.05)$.

\begin{tabular}{|lc|}
\hline Table 1: Baseline Characteristics of the Patients ${ }^{\mathrm{a}, \mathrm{b}}$ & \multicolumn{1}{c|}{ Results } \\
Age & $14.32 \pm 7.01$ \\
Sex, Male & $54(52.4)$ \\
Smoker & $20(19.4)$ \\
Diabetes & $28(27.2)$ \\
Hypertensive & $69(67)$ \\
Ischemic Heart Disease & $66(64.1)$ \\
Normal/Mild CAD & $29(28.1)$ \\
Single Vessel Disease & $20(19.4)$ \\
Two Vessel Disease & $3(2.9)$ \\
Three Vessel Disease & $14(13.6)$ \\
Unknown & $37(35.9)$ \\
Open Heart Surgery & $19(18.4)$ \\
CABG & $12(11.7)$ \\
MVR & $2(1.9)$ \\
AVR & $4(3.9)$ \\
ASD Closure & $1(1)$ \\
Arotic Valve Involvment & $42(42.7)$ \\
Mild & $36(34.9)$ \\
Moderate-Severe & $8(7.8)$ \\
\hline Dan & \\
\hline
\end{tabular}

${ }^{a}$ Data in table are presented as No. (\%) or Mean \pm SD.

${ }^{\mathrm{b}}$ Abbreviations: CAD, coronary artery disease; MVR, mitral valve replacement; AVR, aortic valve replacement; ASD, atrial septum defect; $\mathrm{CABG}$, coronary artery bypass graft.

\begin{tabular}{|lccccc|}
\hline \multicolumn{2}{l}{ Table 2: Prevalance of Conduction Disterbance $(\mathrm{P}=0.180)^{\mathrm{a}, \mathrm{b}}$} & & & \\
& CHB & Second-Degree AV Block & SSS & AV Block + SSS & Others * $^{*}$ \\
HLA-B27 Negtive $(\mathrm{n}=97)$ & $69(71.1)$ & $9(9.3)$ & $5(5.2)$ & $4(4.1)$ & $10(10.3)$ \\
HLA-B27 Positive $(\mathrm{n}=6)$ & $3(50)$ & $0(0)$ & $2(33.3)$ & $0(0)$ & $1(16.7)$ \\
\hline
\end{tabular}

${ }^{\mathrm{a}}$ Data in table are presented as No. (\%) or Mean \pm SD.

${ }^{\mathrm{b}}$ Abbreviations: CHB, complete heart block; AV, atrioventricular; SSS, sick sinus syndrome.

${ }^{*}$ Includeing prolonged HV interval.

Table 3: Prevalance of Aortic Valve Involvement According to HLA-B27 $(\mathrm{P}=0.05)^{\mathrm{a}}$

\begin{tabular}{lcc|} 
& No Arotic Valve Regurgitation & Arotic Valve Regurgitation \\
HLA-B27 Negtive $(\mathbf{n}=\mathbf{9 7})$ & $58(59.8)$ & $39(40.2)$ \\
HLA-B27 Positive $(\mathbf{n}=\mathbf{6})$ & $1(16.7)$ & $5(83.3)$ \\
\hline
\end{tabular}

${ }^{a}$ Data in table are presented as No. (\%) or Mean \pm SD. 


\section{DISCUSSION}

Previous studies showed that HLA-B27 was positive in $15-20 \%$ of Patients who had pacemakers (8). In our study, positive HLA-B27 test was less frequent and was only seen in $6(5.8 \%)$ patients. The prevalence of HLA-B27 positivity is lower in the Iranian population compared to many other countries, and this may explain the lower frequency of HLA-B27 in our study. In a previous study, Adib et al. found that the prevalence of HLA-B27 in the healthy Iranian population was about $8.6 \%$ [4]. The overall frequency of HLA-B27 in the Iranian society has been reported to be 2.5 to $8.6 \%$ [3-5].

About $2-10 \%$ of patients with AS have some kind of heart involvement. On the other hand, it has been approved that patients with AS have not only aortic involvement, but also increased risk of conduction defects, valvular diseases and cardiomyopathy [8].

Increased prevalence of cardiac pacemaker implantation in young males with AS and other rheumatologic diseases associated with HLA-B27 theorized that perhaps the HLA-B27 alone and not in the form of rheumatic disease is associated with conduction disorders. Expression of HLA-B27 in patients with AS may be responsible for cardiac involvement [9, 10]. Bergfeldt et al. evaluated that cardiac conduction disorders might be associated with HLA-B27 alone or not [11]. Bergfeldt et al. found out that permanent pacemaker implantation had a higher prevalence in patients with positive HLA-B27 compared with seronegative arthritis patients. They showed that significantly more complete heart block occurs as the indication of pacemaker implantation in patients with HLA-B27 [11]. In our study as well as Bergfeldt's study, complete heart block was higher as the indication of pacemaker, although this finding was not significant in our study.

Brags-Armas and his colleagues observed that $5 \%$ of patients undergoing cardiac pacemaker had HLA-B27; however, in their study, positive HLA-B27 was not much prevalent in patients with permanent pacemakers compared with a control group without pacemaker [12].

In a study published in 1983, Bergfeldt et al. stated that the pathophysiology of AV block may be the same as AS. In an electrophysiological study of 12 patients with HLA-B27-associated diseases and complete heart block, they found that these patients had predominantly suprahisian location of AV block and also their conductive systems were diffusedly affected. They suggested that HLA-B27 was one of the causes of high-grade AV block [8]. In our study, three out of six patients had AV block and one had fascicular block. However, in our study, no significant difference was observed between types of conduction disturbances.

Bergfeldt found that the frequency of HLA-B27 was higher in pacemaker-treated males in contrast with females and suggested a gender-linked difference in disease susceptibility [13]. The positivity of HLA-B27 was not significantly different in both genders in our study which may be due to our small sample size.

There have been two prevalent theories about the etiology of conduction disturbances in patients with AS: inflammation in the intraventricular septum, leading to damage and anomalies in the AV nodal artery, leading to AV node dysfunction. The pathophysiological explanation for the role of HLA-B27 and conduction disturbances is not completely understood. Anomalies in the AV nodal artery or AV node damage due to inflammation of intraventricular septum may be associated with AV nodal conduction disturbance in patients with positive HLA-B27, especially in patients with AS [14].

\section{ACKNOWLEDGEMENTS}

None declared.

\section{CONFLICTS OF INTERESTS}

There is no conflict of interest.

\section{FUNDING}

Authors declared there is no funding or support.

\section{REFERENCES}

1. Mann DL, Zipes DP, Libby P, Bonow RO, Braunwald E. Braunwald's heart disease : a textbook of cardiovascular medicine. 10th ed2015. 2. Garcia-Medel N, Sanz-Bravo A, Alvarez-Navarro C, Gomez-Molina P, Barnea E, Marcilla M, et al. Peptide handling by HLA-B27 subtypes influences their biological behavior, association with ankylosing spondylitis and susceptibility to endoplasmic reticulum aminopeptidase 1 (ERAP1). Mol Cell Proteomics. 2014;13(12):3367-80. DOI: $10.1074 / \mathrm{mcp} . M 114.039214$ PMID: 25187574

3. Alavi SM, Seraj MS, Etemadi A, Moradzadegan H. Frequency of HLA-B27 antigen in Iranian patients with brucellosis and it's relationship with osteoarticular complication. Pak J Med Sci April-June. 2009;25(2):253-6.

4. Adib KM, Hosseini H. Association of HLA_B27 with ankylosing spondylitis in Isfahan, Iran. Med J Islamic Repub Iran. $1994 ; 8(3): 159-61$.

5. Khan MA. HLA-B27 and its pathogenic role. J Clin Rheumatol. 2008;14(1):50-2. DOI: 10.1097/RHU.0b013e3181637a38 PMID: 18431102

6. Vinsonneau U, Brondex A, Mansourati J, Saraux A, Cornily JC, Arles F, et al. Cardiovascular disease in patients with spondyloarthropathies. Joint Bone Spine. 2008;75(1):18-21. DOI: 10.1016/j. jbspin.2007.04.011 PMID: 17913549

7. Reveille JD, Ball EJ, Khan MA. HLA-B27 and genetic predisposing factors in spondyloarthropathies. Curr Opin Rheumatol. 2001;13(4):265-72. PMID: 11555726

8. Bergfeldt L, Vallin H, Edhag O. Complete heart block in HLA B27 associated disease. Electrophysiological and clinical characteristics. Br Heart J. 1984;51(2):184-8. DOI: 10.1136/hrt.51.2.184 PMID: $\underline{6607060}$

9. Bergfeldt L. HLA B27-associated rheumatic diseases with severe cardiac bradyarrhythmias. Clinical features and prevalence in 223 men with permanent pacemakers. Am J Med. 1983;75(2):210-5. DOI: 10.1016/0002-9343(83)91193-2 PMID: 6603791

10. Moyssakis I, Gialafos E, Vassiliou VA, Boki K, Votteas V, Sfikakis PP, et al. Myocardial performance and aortic elasticity are impaired in patients with ankylosing spondylitis. Scand J Rheumatol. 2009;38(3):216-21. DOI: $10.1080 / 03009740802474672$ PMID: 19229673

11. Bergfeldt L, Moller E. Complete heart block--another HLA B27 associated disease manifestation. Tissue Antigens. 1983;21(5):38590. DOI: $10.1111 /$ j.1399-0039.1983.tb00188.x PMID: 6868058

12. Bruges-Armas J, Lima C, Simas Lopes D, Schneider V, Paisana Lopes JP, Ferreira Gomes A, et al. HLA-B27 in patients with a permanent pacemaker. Ann Rheum Dis. 2003;62(10):1018. DOI: 10.1136/ ard.62.10.1018 PMID: 12972488

13. Bergfeldt L, Moller E. Pacemaker treated women with heart block have no increase in the frequency of HLA-B27 and associated rheumatic disorders in contrast to men--a sex linked difference in disease susceptibility. J Rheumatol. 1986;13(5):941-3. PMID: 3493346

14. Momeni M, Taylor N, Tehrani M. Cardiopulmonary manifestations of ankylosing spondylitis. Int J Rheumatol. 2011;2011. DOI: 10.1155/2011/728471 PMID: 21547038 